\section{Global and Semi-Global Stabilizability in Certain Cascade Nonlinear Systems}

\author{
J. H. Braslavsky and R. H. Middleton
}

Abstract-This paper addresses the issue of global and semi-global stabilizability of an important class of nonlinear systems, namely, a cascade of a linear, controllable system followed by an asymptotically (even exponentially) stable nonlinear system. Such structure may arise from the normal form of "minimum phase" nonlinear systems that can be rendered input-output linear by feedback. These systems are known to be stabilizable in a local sense, and, in some cases, global stabilizability results have also been obtained. It is also known, however, that when the linear "connection" to the nonlinear system is nonminimum phase, i.e., it has zeros with positive real part, then global or semi-global stabilizability may be impossible. Indeed, it has been shown that for any given nonminimum phase linear subsystem, there exists an asymptotically stable nonlinear subsystem for which the cascade cannot be globally stabilized. We expand on the understanding of this area by establishing, for a broader class of systems, conditions under which global or semiglobal stabilization is impossible for linear and nonlinear feedbacks.

\section{INTRODUCTION}

The stabilizability of interconnected nonlinear systems has attracted increasing interest since the appearance in the control scene of the normal form and zero dynamics [1]. A number of recent papers have considered the problem of global stabilization of linear-nonlinear cascade systems of the form

$$
\begin{aligned}
& \dot{x}=f_{0}(x)+f(x, \xi, y), \quad x \in \mathbf{R}^{l} \\
& \dot{\xi}=A \xi+B u, \quad \xi \in \mathbf{R}^{n}, \quad u \in \mathbf{R}^{m} \\
& y=C \xi, \quad y \in \mathbf{R}^{p}
\end{aligned}
$$

where $f_{0}$ and $f$ are smooth (i.e., $C^{\infty}$ ) functions, $\dot{x}=f_{0}(x)$ is globally asymptotically stable (GAS) at $x=0, f(x, 0,0)=0$, and the linear subsystem $(A, B, C)$ is assumed to be stabilizable by state feedback.

Although these systems are asymptotically stabilizable by linear $\xi$-feedback in a local sense (e.g., [2]), the extension of such results to being global is not immediate, and further conditions may need to be applied. For example, it is known that the cascade (1) is not necessarily globally stabilizable by linear $\xi$-feedback only, i.e., with $u=K \xi$, due to the existence of phenomena like peaking [3], [4]. Another obstacle to global stabilizability has been recently identified as unboundedness unobservability [5], where some unmeasured states may escape in finite time while the output remains bounded.

In [6] and [7], sufficient conditions have been given to globally asymptotically stabilize these "partially linear composite systems" by a smooth feedback $u=K \xi+v(x, \xi)$. A key restriction on the cascades for which GAS can be achieved is that either the linear subsystem must be weakly minimum phase [6]-[10], or the growth of the connection terms is constrained [7, Proposition 5].

The conditions for GAS mentioned above are only sufficient but, as it has been pointed out [3], they are somehow close to being necessary. Furthermore, in [7] it has been proven that given a nonminimum phase linear system, one can always find a nonlinear system such that the cascade verifying all the other conditions is not globally stabilizable. The results in this paper further expand on this

Manuscript received August 4, 1994; revised January 20, 1995 and December 4,1995 .

The authors are with the Department of Electrical and Computer Engineering, The University of Newcastle, NSW 2308, Australia.

Publisher Item Identifier S 0018-9286(96)03711-7. idea by establishing, for a broader class of systems, conditions under which GAS is impossible.

In addition to GAS, we consider the problem of semi-global stabilizability.' The property of semi-global stabilizability has been studied in many recent papers on nonlinear systems [2], [3], [11]-[13]. A system is semi-globally stabilizable to an equilibrium point $x_{c}$ by means of a class $\mathcal{F}$ of feedback control laws if, for any a priori determined compact set $\Omega$ of initial conditions, there exists a control law in $\mathcal{F}$ that makes $x_{e}$ asymptotically stable with a domain of attraction that contains $\Omega$.

In this paper, we show that a given class of GAS nonlinear systems cascaded with any single-input/single-output (SISO) nonminimum phase linear system is not (even semi-) globally stabilizable by linear, time-invariant, static $\xi$-feedback. In addition, we give a class of SISO linear-nonlinear cascades systems for which no control can give global or semi-global stability. These results are illustrated by an example, where a system of the first class is semi-globally stabilized by linear time-varying $\xi$-feedback, and globally stabilized by nonlinear $(\xi, x)$-feedback.

Notation: The symbols $\mathbf{R}, \mathbf{C}$, denote real and complex numbers, respectively, and $\operatorname{Re}(x)$ denotes the real part of the number $x . \mathbf{C}^{-}$ and $\mathrm{C}^{+}$are the open left- and right-half planes, respectively. $\|\cdot\|$ is the Euclidean norm for matrices. The rest of the notation is fairly standard, or introduced where required.

\section{AN EXPLosive CASCADE}

We study the following class of linear-nonlinear cascades

$$
\begin{aligned}
\dot{x} & =-\alpha x+\beta x^{m} y^{2 p} \\
\dot{\xi} & =A \xi+B u \\
y & =C \xi+D u
\end{aligned}
$$

where $m, p$ are positive integers, $m>1 ; \alpha, \beta$ are positive real numbers; $x, u, y \in \mathbf{R}$, and $\xi \in \mathbf{R}^{n}$. We assume that the pair $(A, B)$ is controllable and the linear subsystem (3), (4) is nonminimum phase, i.e., its transfer function, $\mathcal{G}(s)=C(s I-A)^{-1} B+D$, has a zero at the complex frequency $s=\nu$, where $\operatorname{Re}(\nu)>0$. We also assume $C \neq 0$.

These cascades present two fundamental characteristics that make difficult their stabilizability in a global sense: 1) the linear "connection" (3), (4) is nonminimum phase, and 2) the nonlinear subsystem (2) has a strong coupling with its input. The nonminimum phase feature implies the existence of peaking in $y$ when high gain $\xi$ feedback is used. On the other hand, the strong coupling of the nonlinear subsystem implies that if $y$ is not sufficiently small, finite time escape of $x$ may occur. Therefore, even when the nonlinear subsystem is globally exponentially stable without input, the cascade may fail to be GAS.

Let us now characterize a necessary condition for the semi-global stabilizability of system (2)-(4) that will be used later. Define the change of coordinates

$$
\eta(t) \doteq \frac{e^{-\tilde{\alpha} t}}{(m-1) x^{m-1}(t)}, \quad x \neq 0
$$

with $\tilde{\alpha} \doteq \alpha(m-1)$. Then, we can write (2) as

$$
\dot{\eta}=-\beta y^{2 p} e^{-\tilde{\alpha} t}
$$

\footnotetext{
${ }^{1}$ Some authors use the terms potentially global or on compacta stabilizabil-
} 
to which corresponds the solution

$$
\eta(t)=\eta(0)-\beta \int_{0}^{t} e^{-\bar{\alpha} \tau} y^{2 p}(\tau) d \tau
$$

Suppose that $x_{0}$ is a given positive initial condition for (2). Then $\eta(0)=x_{0}^{1-m} /(m-1)$ is also positive and so, from (6), we see that $\eta(t)$ may get arbitrarily close to zero at any $t$ for which the integral on the right-hand side (RHS) of (6) is large enough, eventually resulting in the escape to infinity of the nonlinear state $x(t)$. Thus, a necessary condition for the stability of (2) with any positive initial condition is that the inequality

$$
\int_{0}^{t} e^{-\tilde{\alpha} \tau} y^{2 p}(\tau) d \tau<\frac{\eta(0)}{\beta}
$$

be satisfied for any $t \geq 0$, or equivalently, since $\eta(t)$ is monotonic

$$
\int_{0}^{\infty} e^{-\tilde{\alpha} \tau} y^{2 p}(\tau) d \tau<\frac{\eta(0)}{\beta}, \quad \forall \eta(0)>0 .
$$

\section{Does a Nonminimum Phase Linear SUBSYSTEM NECESSARILY IMPLY PEAKING?}

Condition (7) is our starting point to explore a key connection between the "degree of nonminimum phaseness" of the linear subsystem and the dynamics of the nonlinear subsystem. In this section, we shall see that this connection determines the stabilizability properties of the cascade (2)-(4). More specifically, we shall demonstrate 1) conditions under which the cascade cannot be semi-globally stabilizable and 2) conditions under which the cascade cannot be semi-globally stabilized by using linear, time-invariant, static feedback of the linear subsystem states. We shall also extend these issues to a more general class of cascades, where the nonlinear subsystem presents a particular type of Lyapunov function.

We require some preliminary results on linear systems and matrices. The following lemma recalls an input-output relation satisfied by linear systems with nonminimum phase zeros [14].

Lemma 1: Consider a linear system

$$
\begin{aligned}
& \dot{\xi}=A \xi+B u \\
& y=C \xi+D u
\end{aligned}
$$

with $\xi$ in $\mathbf{R}^{n}$ and $u, y$ in $\mathbf{R}$. If $\nu$ is a nonminimum phase zero of the transfer function $\mathcal{G}(s)=C(s I-A)^{-1} B+D$, and $y(t)$ is the bounded response to a bounded input $u(t)$ with initial condition $\xi_{0}$, then

$$
\begin{aligned}
\mathcal{Y}(\nu) & =\int_{0}^{\infty} e^{-\nu \tau} y(\tau) d \tau \\
& =C(\nu I-A)^{-1} \xi_{0} .
\end{aligned}
$$

Proof: The proof follows trivially on noting that since $y(t)$ and $u(t)$ are bounded, $s=\nu$ is in the region of convergence of their corresponding Laplace transforms, $\mathcal{Y}(s)$ and $\mathcal{U}(s)$.

The next theorem states conditions under which the cascade (2), (3) cannot be stabilized in a global sense.

Theorem 2: The cascade system (2), (3) is not semi-globally stabilizable if the linear subsystem (3) has a nonminimum phase zero at $s=\nu$ such that $\operatorname{Re}(\nu)>\alpha(m-1) / 2 p$.

Proof: From Lemma 1 we can write

$$
\left|C(\nu I-A)^{-1} \xi_{0}\right| \leq \int_{0}^{\infty} e^{-\operatorname{Re}(\nu) \tau}|y(\tau)| d \tau .
$$

By applying Hölder's inequality to the RHS of (8) and solving an integral, we obtain

$$
\int_{0}^{\infty} e^{-\operatorname{Re}(\nu) \tau}|y(\tau)| d \tau \leq
$$

$$
\left[\frac{2 p-1}{2 p \operatorname{Re}(\nu)-\tilde{\alpha}}\right]^{(2 p-1) / 2 p}\left[\int_{0}^{\infty} e^{-\tilde{\alpha} \tau} y^{2 p}(\tau) d \tau\right]^{1 / 2 p}
$$

where, as in Section II, we denoted $\tilde{\alpha}=\alpha(m-1)$. Inequalities (8) and (9) give

$$
\int_{0}^{\infty} e^{-\tilde{\alpha} \tau} y^{2 p}(\tau) d \tau \geq M
$$

where

$$
M=\left[\frac{2 p \operatorname{Re}(\nu)-\tilde{\alpha}}{2 p-1}\right]^{2 p-1}\left|C(\nu I-A)^{-1} \xi_{0}\right|^{2 p} .
$$

Note that $M$ is a number independent of any feedback. Moreover, $M$ can always be made positive and arbitrarily large by appropriate choice of the initial condition $\xi_{0}$. As we have seen in Section II, the stability of the cascade in a global sense requires that (7) be true for all initial conditions. Yet, (10) and the previous remark on $M$ indicate that given $x_{0}$, there always exist values of $\xi_{0}$ for which (7) is violated. The result then follows.

The above theorem tells us that if the linear subsystem has a nonminimum phase zero relatively "fast" with respect to the dynamics of the nonlinear subsystem, namely,

$$
\boldsymbol{\operatorname { R e }}(\nu)>\frac{\alpha(m-1)}{2 p}
$$

then the cascade cannot be globally or semi-globally stabilized by any control. At this point, a natural question arises: is it possible to achieve GAS or semi-GAS if (11) is not satisfied? As we see next, the answer to this question is again negative if we can only use linear, time invariant, static $\xi$-feedback.

Theorem 3: If the linear subsystem (3) is nonminimum phase, then the cascade system (2), (3) is not semi-globally stabilizable by linear, time-invariant, static $\xi$-feedback, that is, $u=K \xi$, with $K$ in $\mathbf{R}^{1 \times n}$.

Before proving Theorem 3 , we require a preliminary result on linear systems. Let $(A, B)$ be a controllable pair, $A$ in $\mathbf{R}^{n \times n}, B$ in $\mathbf{R}^{n \times 1}$. Denote by $\mathcal{K}$ the set of all row vectors $K$ in $\mathbf{R}^{1 \times n}$ such that $A_{K} \doteq A+B K$ has all its eigenvalues in $\mathbf{C}^{-}$. Then, we have the following lemma.

Lemma 4: If $\nu$ is a complex number with positive real part, then

$$
\sup _{K \in \mathcal{K}}\left\|\left(\nu I-A_{K}\right)^{-1}\right\|<\infty .
$$

Proof: Although perhaps intuitively reasonable, this proof is a little long and involved, and has been omitted due to space limitations. See [15] for further details.

Remark 1: Note that the above result relies on the fact that the linear system associated with $(A, B)$ is single-input. Indeed, Lemma 4 is not true for multi-input systems, as can be seen in the following simple counterexample. Consider $A=0^{2 \times 2}, B=I^{2 \times 2}$, and choose

$$
K=\left[\begin{array}{cc}
-\rho_{1} & 0 \\
-\rho_{2} & -\rho_{3}
\end{array}\right]
$$

with $\rho_{i}$, positive numbers. Then write

$$
\begin{aligned}
\left(\nu I-A_{K}\right)^{-1} & =(\nu I-K)^{-1} \\
& =\left[\begin{array}{cc}
\left(\nu+\rho_{1}\right)^{-1} & 0 \\
\rho_{2}\left[\left(\nu+\rho_{1}\right)\left(\nu+\rho_{3}\right)\right]^{-1} & \left(\nu+\rho_{3}\right)^{-1}
\end{array}\right] .
\end{aligned}
$$

Notice that the stability of $A_{K}$ is independent of $\rho_{2} .\left\|\left(\nu I-A_{K}\right)^{-1}\right\|$, however, grows without bound with $\rho_{2}$. 
Proof of Theorem 3: Suppose we stabilized the linear subsystem (3), (4) with a state feedback $u=K \xi$, so that all the eigenvalues of the closed-loop evolution matrix, $\lambda_{i}\left\{A_{K}\right\}$, with $i=1,2, \ldots, n$, are in $\mathbf{C}^{-}$. Let $\nu$ be a nonminimum phase zero of the linear subsystem, let $\mathcal{Y}(s)$ be the Laplace transform of the closed-loop output, and $\tilde{\alpha}$ as defined just before (5). Then, we can write

$$
\begin{aligned}
\mathcal{Y}(\tilde{\alpha}) & =(C+D K)\left(\tilde{\alpha} I-A_{K}\right)^{-1} \xi_{0} \\
& =(C+D K)[\nu I-A+(\tilde{\alpha}-\nu) I-B K]^{-1} \xi_{0} .
\end{aligned}
$$

By applying the matrix inversion lemma to the last equation, we get

$$
\begin{aligned}
\mathcal{Y}(\tilde{\alpha})= & (C+D K)(\nu I-A)^{-1} \\
& \cdot\left\{I-H\left[I+(\nu I-A)^{-1} H\right]^{-1}(\nu I-A)^{-1}\right\} \xi_{0}
\end{aligned}
$$

where $H=(\tilde{\alpha}-\nu) I-B K$. Using the fact that $\mathcal{G}(\nu)=D+C(\nu I-$ $A)^{-1} B=0$, and after some manipulation, we obtain

$$
\mathcal{Y}(\tilde{\alpha})=C(\nu I-A)^{-1}\left[I-(\tilde{\alpha}-\nu)\left(\tilde{\alpha} I-A_{K}\right)^{-1}\right] \xi_{0} .
$$

Now suppose that we choose the initial condition $\xi_{0}$ proportional to the complex conjugate transpose of the row vector $C(\nu I-A)^{-1}$, i.e.,

$$
\xi_{0}=\gamma\left(\bar{\nu} I-A^{T}\right)^{-1} C^{T}
$$

with $\gamma$ a real number. Notice that the vector $\left(\bar{\nu} I-A^{T}\right)^{-1} C^{T}$ is nonzero, since $(\nu I-A)^{-1}$ is full-rank and $C \neq 0$. Let $\Gamma$ denote the matrix $I-(\tilde{\alpha}-\nu)\left(\tilde{\alpha} I-A_{K}\right)^{-1}$. Then, substituting (15) in (14) and taking absolute values on both sides gives

$$
\begin{aligned}
|\mathcal{Y}(\tilde{\alpha})| & =|\gamma|\left|C(\nu I-A)^{-1} \Gamma\left(\bar{\nu} I-A^{T}\right)^{-1} C^{T}\right| \\
& \geq|\gamma|\left\|C(\nu I-A)^{-1}\right\|^{2} \underline{\sigma}
\end{aligned}
$$

where $\underline{\sigma}$ denotes the smallest singular value of the matrix $\Gamma$. Then we have

$$
\begin{aligned}
\underline{\sigma} & =\left\|\Gamma^{-1}\right\|^{-1} \\
& =\left\|\left(\nu I-A_{K}\right)^{-1}\left(\tilde{\alpha} I-A_{K}\right)\right\|^{-1} \\
& =\left\|I+(\tilde{\alpha}-\nu)\left(\nu I-A_{K}\right)^{-1}\right\|^{-1} \\
& \geq \frac{1}{1+|\tilde{\alpha}-\nu|\left\|\left(\nu I-A_{K}\right)^{-1}\right\|} .
\end{aligned}
$$

Lemma 4 assures that $\left\|\left(\nu I-A_{K}\right)^{-1}\right\|$ is uniformly bounded on $\mathcal{K}$; denote this bound by $\mu$. Hence (16) and (17) yield

$$
|\mathcal{Y}(\hat{\alpha})| \geq \frac{|\gamma|\left\|C(\nu I-A)^{-1}\right\|^{2}}{1+|\tilde{\alpha}-\nu| \mu} .
$$

On the other hand, we can bound $|\mathcal{Y}(\tilde{\alpha})|$ and use Hölder's Inequality to get

$$
\begin{aligned}
|\mathcal{Y}(\tilde{\alpha})| & \leq \int_{0}^{\infty} e^{-\bar{\alpha} \tau}|y(\tau)| d \tau \\
& \leq\left(\frac{1}{\tilde{\alpha}}\right)^{(2 p-1) / 2 p}\left[\int_{0}^{\infty} e^{-\bar{\alpha} \tau} y^{2 p}(\tau) d \tau\right]^{1 / 2 p} .
\end{aligned}
$$

Combining (18) and (19) renders

$$
\int_{0}^{\infty} e^{-\tilde{\alpha} \tau} y^{2 p}(\tau) d \tau \geq M
$$

where

$$
M=\frac{(\tilde{\alpha})^{2 p-1}|\gamma|^{2 p}|| C(\nu I-A)^{-1} \|^{4 p}}{(1+|\tilde{\alpha}-\nu| \mu)^{2 p}} .
$$

Here, as in the proof of Theorem $2, M$ is a number independent of the feedback gain $K$. Moreover, $M$ can be set positive and arbitrarily large by selection of the number $\gamma$. Hence, the necessary condition for stability (7) can always be violated, and the result follows.

The issues addressed by Theorems 2 and 3 can be extended to cascaded linear-nonlinear systems with the nonlinear subsystem having a particular type of Lyapunov function.
Theorem 5: Consider the system

$$
\begin{aligned}
\dot{x} & =f(x, y) \\
\dot{\xi} & =A \xi+B u \\
y & =C \xi+D u
\end{aligned}
$$

where the linear subsystem is nonminimum phase, i.e., has a zero $\nu \in \mathrm{C}^{+}$, and the nonlinear subsystem $\dot{x}=f(x, 0)$ has a Lyapunov function $V(x) \geq 0, V(x)=0 \Leftrightarrow x=0$, and $V(x) \rightarrow \infty \Rightarrow\|x\| \rightarrow$ $\propto$, such that

$$
\dot{V} \geq-\alpha V+\beta V^{n} y^{2 p}
$$

with $\alpha, \beta$ positive real numbers, and $n, p$ positive integers, $n>1$. Then:

1) The cascade (20) is not semi-globally stabilizable by linear, time-invariant, static $\xi$-feedback.

2) If $\operatorname{Re}(\nu)>\alpha(m-1) / 2 p$, the cascade (20) is not semi-globally stabilizable at all.

Proof: Note that if $V$ and $W$ satisfy

$$
\begin{aligned}
\dot{V} & \geq-\alpha V+\beta V^{n} y^{2 p} \\
\dot{W} & =-\alpha W+\beta W^{n} y^{2 p}
\end{aligned}
$$

and $V(t=0)=W(t=0)$, then $V \geq W$ for all $t>0$; see for example [16]. The proof then follows by the same arguments used in the proofs of Theorems 2 and 3 applied to the cascade

$$
\begin{aligned}
\dot{W} & =-\alpha W+\beta W^{n} y^{2 p} \\
\dot{\xi} & =A \xi+B u \\
y & =C \xi+D u .
\end{aligned}
$$

To illustrate these results, the next section presents an example of a system for which $\operatorname{Re}(\nu) \leq \alpha(m-1) / 2 p$, i.e., which we could expect to globally stabilize, although-recalling Theorem 3-not using a feedback $u=K \xi$. As we shall see, we can achieve semiglobal stability using linear static time-varying $\xi$-feedback and global stability using nonlinear $(\xi, x)$-feedback.

\section{EXAMPle: A DyNAMic "Gambit"}

As seen in the previous section, the question of the linear subsystem being nonminimum phase is critical to the stabilizability of these cascades, particularly if we are restricted to a control law $u=K \xi$. Nevertheless, the situation is different if we use a linear timevarying feedback $u=K(t) \xi$, or a nonlinear $(\xi, x)$-feedback. More specifically, in this section we present an example where the nonminimum phase zeros of the linear subsystem are "not too fast" (see Theorem 2), and so we obtain global and semi-global stability using a switching control strategy.

The proposed switching strategy consists of two stages. Firstly, annihilate the output of the linear subsystem to let the nonlinear state go to zero, while allowing the linear states to grow exponentially. Then, after a suitable period of time, switch to a control strategy that stabilizes the linear states. Since the linear states grow at a slower rate than the decay of the nonlinear state, it is always possible to find a switching time avoiding finite time escape of $x$. This stratagem is somehow reminiscent of a chess gambit, where a piece (stability of the linear states) has to be initially sacrificed to ultimately win the match (global stability of the cascade).

We now introduce the example. Consider the cascade

$$
\begin{aligned}
& \dot{x}=-3 x+x^{2} y^{2} \\
& \dot{\xi}=u \\
& y=\xi-u
\end{aligned}
$$


where $x, y, u$ are in $\mathbf{R}$. The linear subsystem has a nonminimum phase zero $\nu=1$. Hence, by Theorem 3 , we know that linear static $\xi$-feedback cannot-in a global sense-stabilize the cascade. Also, $\nu<\tilde{\alpha} / 2 p=3 / 2$. We show next how the cascade (22) is semiglobally stabilized using linear time-varying $\xi$-feedback, and later on, how global stability is achieved by nonlinear $(\xi, x)$-feedback. The following remark is first in order.

Remark 2: Note that $x=0$ is an invariant manifold of system (22) independently of the control law. Hence, if at any time, $T$ say, $x(T)>0[x(T)<0]$, then for $t>T, x(t) \geq 0[x(t) \leq 0]$. This will allow us to analyze separately the cases $x(\bar{T})>0$ and $x(T)<0$.

\section{A. Semi-Global Stability}

Let the initial conditions be $x_{0}, \xi_{0}$. We propose the following control strategy:

$$
u=\left\{\begin{array}{cl}
-\xi & \text { if } x_{0} \leq 0, \text { or if } x_{0}>0 \text { and } t \geq T \\
\xi & \text { if } x_{0}>0 \text { and } t<T
\end{array}\right.
$$

where

$$
T=\log ^{+}\left(\frac{4 \xi_{0}^{2} x_{0}}{5}\right)+\epsilon .
$$

Here $\log ^{+}(\cdot)$ denotes $\max \{\log (\cdot), 0\}$, and $\epsilon$ is a small positive number. This control law is fixed if $x_{0} \leq 0$, and has at most one switching at $t=T$ if $x_{0}>0$.

Case A.1: $\left(x_{0} \leq 0\right)$ If $x_{0} \leq 0$, then the control law $u=-\xi$ gives $u, \xi, x$ bounded for $t \geq 0$ and, moreover, $u(t), \xi(t), x(t) \rightarrow$ 0 as $t \rightarrow \infty$.

Proof: The differential equations become

$$
\begin{aligned}
& \dot{x}=-3 x+x^{2} y^{2} \\
& \dot{\xi}=-\xi \\
& y=2 \xi .
\end{aligned}
$$

Recall that, by Remark $2, x_{0} \leq 0$ implies that $x(t) \leq 0$ for $t>0$. Let $V=-x+\xi^{2} / 2$ be a Lyapunov function candidate for (25) with $x \leq 0$. Then, $\dot{V}=3 x-x^{2} y^{2}-\xi^{2}$, and so $\dot{V} \leq 0$ for $x \leq 0$ and any $\xi$, and $\dot{V}=0$ if and only if $x=0$ and $\xi=0$. Hence, the origin is an asymptotically stable equilibrium for system (25) with initial conditions $x_{0}<0, \xi_{0}$.

Case A.2: $\left(x_{0}>0\right)$ If $x_{0}>0$, then the switching control law given in (23) gives $u, \xi, x$ bounded for $t \geq 0$ and, moreover, $u(t), \xi(t), x(t) \rightarrow 0$ as $t \rightarrow \infty$.

Proof: First, for $t<T$, the control is set to $u=\xi$, so the system has the form

$$
\begin{aligned}
& \dot{x}=-3 x \\
& \dot{\xi}=\xi
\end{aligned}
$$

so $x(t)=e^{-3 t} x_{0}$ and $\xi(t)=e^{t} \xi_{0}$. This obviously makes the linear subsystem unstable, but note that the condition $\nu<\tilde{\alpha} / 2 p$ assures that $x(t)$ goes to zero at a faster rate than that at which $|\xi(t)|$ increases.

Then, at $t=T$-and for all $t>T$ - set the control to $u=-\xi$ to render the linear subsystem stable. The linear state will evolve as $\xi(t)=e^{-(t-2 T)} \xi_{0}$, and the output, $y=2 \xi$, will display a big peak before starting to decrease. Let us see that, by the choice of $T$, this peak does not provoke finite-time escape of $x$ and, furthermore, gives exponential decay of all variables.

Recalling the change of coordinates introduced in Section II, we have the associated variable $\eta(t)=e^{-3 t} / x(t)$, and for $t \geq T$ we can write

$$
\begin{aligned}
\eta(t) & =x_{0}^{-1}-\frac{4}{5} \xi_{0}^{2} e^{4 T}\left(e^{-5 T}-e^{-5 t}\right) \\
& \geq x_{0}^{-1}\left(1-\frac{4}{5} \xi_{0}^{2} x_{0} e^{-T}\right) \\
& \geq x_{0}^{-1}\left(1-e^{-\epsilon}\right)
\end{aligned}
$$

where the last step uses the choice of $T$ in (24). Returning to the original variables, we have obtained

$$
x(t) \leq \frac{e^{-3 t} x_{0}}{1-e^{-\epsilon}}, \quad t \geq T
$$

which shows the boundedness of the nonlinear state for every time $t \geq T$. Since $T$ is finite for finite initial conditions, $\xi$ and $u$ are also exponentially bounded and the result follows.

This strategy can be applied to every given set of initial conditions, thus resulting in semi-global stability. Note that the semi-global property provided by this time-varying controller is not uniform in time, though.

Fig. 1 displays simulation responses for $\xi_{0}=-1, x_{0}=2$, and different switching times. For these initial conditions, (24) gives $T=0.47+\varepsilon$. It can be seen from the plots that the critical case with $\varepsilon=0$ still stabilizes the system, although with a big peak in $x$.

Remark 3: This control strategy for semi-global stabilizability can be generalized to a cascade where the linear subsystem has relative degree greater than zero. In this case, though, we cannot force the linear subsystem output to zero immediately. Instead, we make it approach to zero exponentially rapidly, which is sufficient to avoid finite-time escape of $x(t)$ in a first stage. The determination of a suitable switching time $T$ for ultimate exponential boundedness of all variables follows the same lines given above, but the computation is much more involved. An example with a nonminimum phase linear subsystem of relative degree one can be found in [15]

\section{B. Global Stability}

Alternatively, a nonlinear switching strategy can be devised if we also feed back the nonlinear state $x$. In this case, we can use the control law

$$
u= \begin{cases}\xi & \text { whenever } x \xi^{2} \geq \frac{5 e^{-\varepsilon}}{4}, \\ -\xi & \text { whenever } x \xi^{2}<\frac{5 e^{-\varepsilon}}{4}\end{cases}
$$

with $\varepsilon$ a small positive number. Hence, at most one switching occurs and the system is globally stabilized (and in this case the stability is uniform in time). This is easily seen from the following arguments.

Case B.1: $[x(T) \leq 0]$ if, at any time $t=T$, we have $x(T) \leq 0$, then the control law $u(t)=-\xi(t)$ gives $u, \xi, x$ bounded for $t \geq T$, and moreover, $u(t), \xi(t), x(t) \rightarrow 0$ as $t \rightarrow \infty$.

Proof: The proof follows the same steps in the proof of Case A.1, with $x(T)$ instead of $x_{0}$.

Case B.2: $\left[x(T)>0\right.$ and $\left.x(T) \xi^{2}(T) \leq 5 e^{-\varepsilon} / 4\right]$ if, at any time $t=T$, we have $x(T)>0$ and $x(T) \xi^{2}(T) \leq 5 e^{-\varepsilon} / 4$, then the control law $u=-\xi$ causes $u, \xi, x$ to be bounded for $t \geq T$, and moreover, $u(t), \xi(t), x(t) \rightarrow 0$ as $t \rightarrow \infty$ without further switching.

Proof: For this case, the system's equations are given by (25) again. Let $V=x \xi^{2}$. Then it is easy to check that $\dot{V}=-5 V(1-$ $4 V / 5) \leq-5 V\left(1-e^{-\varepsilon}\right)$, and so $V$ goes to zero exponentially

$$
V(t) \leq V(T) e^{-\delta(t-T)}
$$

with

$$
\delta=5\left(1-e^{-\varepsilon}\right)
$$

i.e., there is no further switching for $t>T$.

The exponential decrease of $V$ determines that also $x$ goes exponentially to zero, since the differential equation for $x$ in (25) can be written as $\dot{x}=-3 x+4 x V$. The solution $x(t)$ is nonnegative for $t \geq T$, by Remark 2 , and more specifically

$$
\begin{aligned}
x(t) & =e^{-3(t-T)} x(T)+\int_{T}^{t} e^{-3(t-r)} x(\tau) V(\tau) d \tau \\
& \leq e^{-3(t-T)} x(T)+\int_{T}^{t} e^{-3(t-\tau)} x(\tau) V(T) e^{-\delta(\tau-T)} d \tau .
\end{aligned}
$$



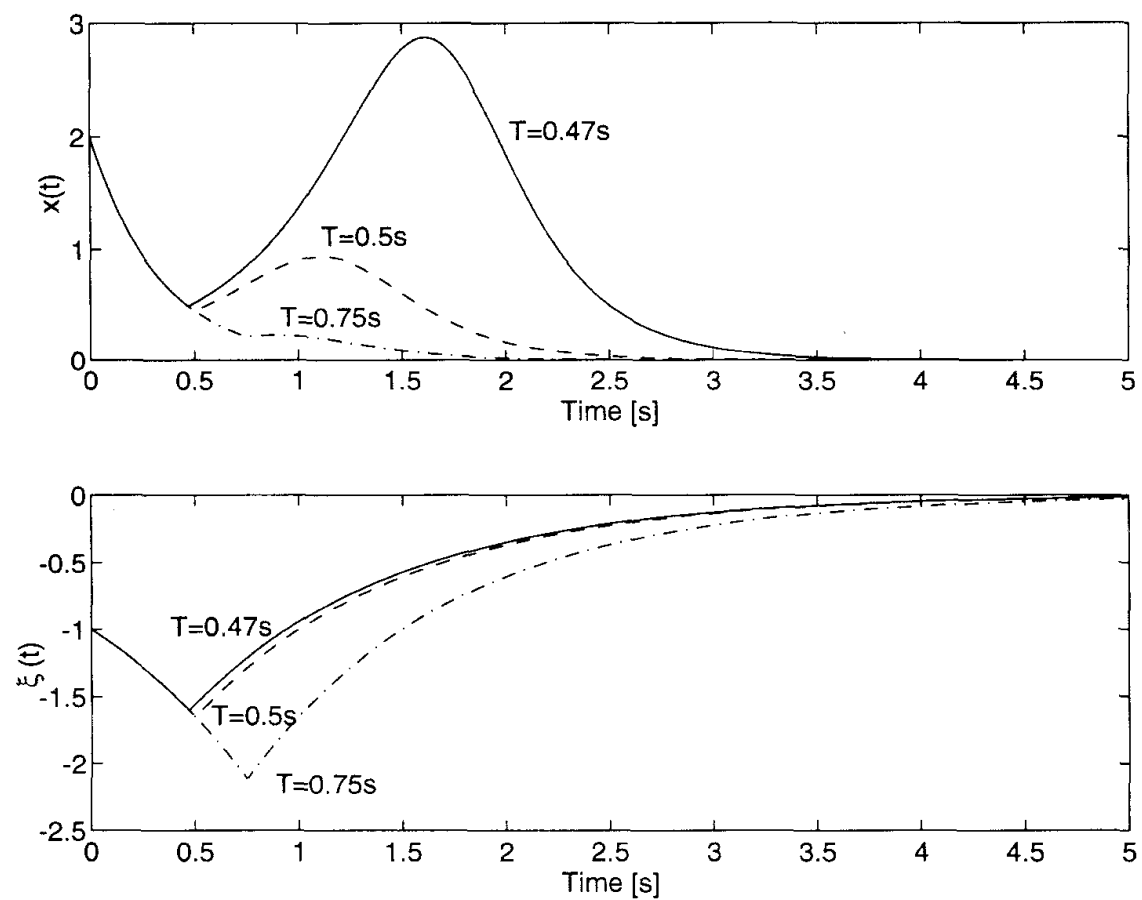

Fig. 1. Time responses for semi-global stability.

Multiplying both sides of the last inequality by $e^{3 t}$, and denoting $z(t)=e^{3 t} x(t)$, we get

$$
z(t) \leq z(T)+e^{\delta T} V(T) \int_{T}^{t} z(\tau) e^{-\delta \tau} d \tau
$$

Applying the Bellman-Gronwall lemma to (29), it follows that

$$
\begin{aligned}
z(t) & \leq z(T) \exp \left[e^{\delta T} V(T) \int_{T}^{t} e^{-\delta \tau} d \tau\right] \\
& \leq z(T) \exp \left[\frac{V(T)}{\delta}\right] .
\end{aligned}
$$

Finally, (30) gives

$$
x(t) \leq e^{-3(t-T)} x(T) \exp \left[\frac{x(T) \xi^{2}(T)}{5\left(1-e^{-s}\right)}\right] .
$$

On the other hand, note that $\xi$ and $u$ are already exponentially decreasing to zero. In effect, with this control law we have that $\xi(t)=\xi(T) e^{-(t-\Upsilon)}=-u(t)$.

Case B.3: $\left[x(T)>0\right.$ and $\left.x(T) \xi^{2}(T)>5 e^{-\varepsilon} / 4\right]$ If, at any time $t=T$, we have $x(T) \xi^{2}(T)>5 e^{-\varepsilon} / 4$, then the control law $u=\xi$ causes $x \xi^{2}$ to decrease exponentially to zero.

Proof: For this case, the system's equations become

$$
\begin{aligned}
& \dot{x}=-3 x \\
& \dot{\xi}=\xi .
\end{aligned}
$$

Then

$$
\begin{aligned}
\frac{d\left(x \xi^{2}\right)}{d t} & =-3 x \xi^{2}+2 x \xi^{2} \\
& =-x \xi^{2}
\end{aligned}
$$

From Cases B.1, B.2, and B.3, we can see that any pair of initial conditions will produce at most one switching of the controller, rendering the system asymptotically stable to zero. Note that in Case

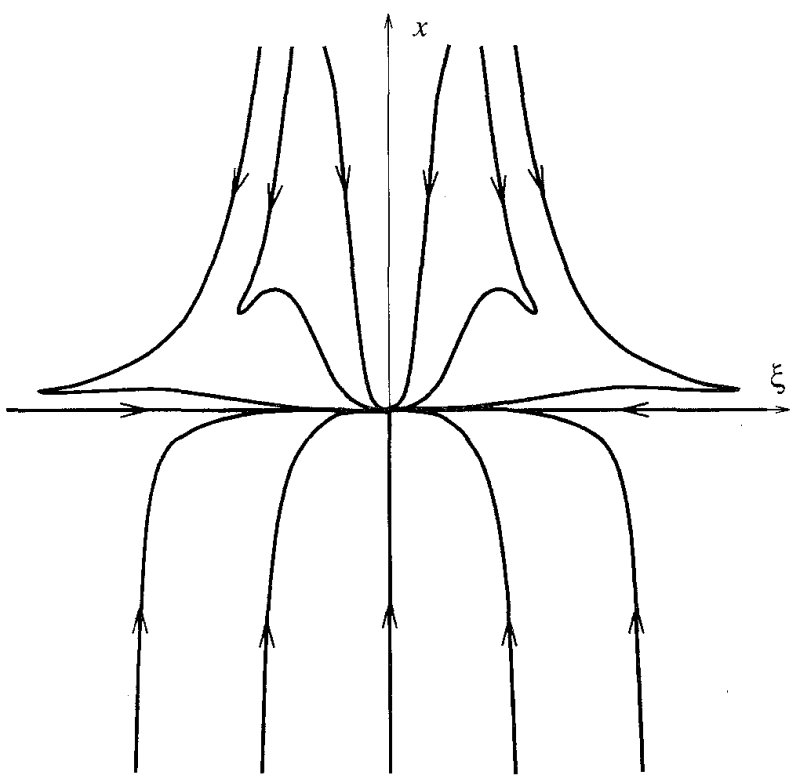

Fig. 2. Phase portrait of the system globally stabilized with nonlinear $(\xi, x)$-feedback

B.3, the linear subsystem will be unstable, $\xi$ growing exponentially, but only for a finite interval of time, namely, until $x(T) \xi^{2}(T) \leq$ $5 e^{-s} / 4$ for some finite $T$. Fig. 2 shows the phase portrait of the globally stable closed-loop system.

Remark 4: We would conjecture that a nonlinear control law can also be generated using these ideas to achieve GAS when the linear subsystem has relative degree greater than zero. Yet, as for the case of semi-global stability, much more involved computations should be expected. 


\section{CONCLUSIONS}

We have considered the problem of global/semi-global stabilization of a class of nonlinear systems in the form of a cascade of a linear, controllable system, and a nonlinear, exponentially stable system. Key features of these systems are: 1) the linear "connection" to the nonlinear system is nonminimum phase, which implies that with highgain $\xi$-feedback, peaking occurs; and 2) the nonlinear system has a strong coupling with its input, which means that if there is sufficient "energy" in the input then a finite time escape may occur.

We have demonstrated families of these cascades for which global/semi-global stabilization by linear time-invariant feedback of $\xi$ is impossible. In addition, we have shown that for systems with "slow" zero dynamics ( $x$ stability) relative to the linear nonminimum phase zero location, even semi-global stabilization is impossible.

By example, we have illustrated linear time-varying and nonlinear feedbacks that can give semi-global/global stabilization for the remaining case, i.e., "fast" zero dynamics. In this case, the approach is somewhat counterintuitive, namely:

1) First, force the input to the nonlinear system to zero using unstable feedback on the linear system. In this case, the nonlinear state $x$ converges to zero rapidly, while the linear state $\xi$ diverges.

2) After a suitable period of time, switch to a linear stabilizing feedback to cause the linear state to return to zero.

This procedure has an interpretation in terms of invertibility of the linear subsystem. Referring to the internal model paradigm [17], an ideal controller would be constructed using the inverse of the plant. When the plant (our linear subsystem) is nonminimum phase, we cannot invert it, as it would render an unstable controller. As our control strategy suggests, however, depending on the relative speed of the nonminimum zero we could invert it "for a while." This period of "disconnection" allows the stabilization of the nonlinear subsystem in a way that, ultimately, the whole cascade remains stable when switching to a stabilizing controller for the linear part.

\section{ACKNOWLEDGMENT}

The authors wish to thank M. Fu and A. Feuer for key suggestions in the development of these results.

\section{REFERENCES}

[1] A. Isidori, Nonlinear Control Systems: An Introduction, 2nd ed. New York: Springer-Verlag, 1989.

[2] C. I. Byrnes and A. Isidori, "Asymptotic stabilization of minimum phase nonlinear systems," IEEE Trans. Automat. Contr., vol. 36, pp. $1122-1137$, Oct. 1991

[3] P. V. Kokotovic, "Joy of feedback: Nonlinear and adaptive," in Proc. 30th IEEE Conf. Decision Control: 199I Bode Prize Lecture, Brighton, England, Dec. 1991

[4] H. J. Sussmann and P. V. Kokotović, "The peaking phenomenon and the global stabilization of nonlinear systems," IEEE Trans. Automat. Contr., vol. 36, pp. 424 440, 1991.

[5] F. Mazenc, L. Praly, and W. P. Dayawansa, "Global stabilization by output feedback: Examples and counter-examples," Syst. Contr. Lett., vol. 23 , pp. 119-125, 1994.

[6] P. V. Kokotović and H. J. Sussmann, "A positive real condition for global stabilization of nonlinear systems," Syst. Conir. Lett., vol. 13, pp. 125-133, 1989.

[7] A. Saberi, P. V. Kokotović, and H. J. Sussmann, "Global stabilization of partially linear composite systems," SIAM J. Contr. Opt., vol. 28, no. 6, pp. 1491-1503, 1990.
[8] R. Ortega, "Passivity properties for stabilization of cascaded nonlinear systems," Automatica, vol. 27, no. 2, pp. 423-424, 1991.

[9] C. I. Byrnes, A. Isidori, and J. C. Willems, "Passivity, feedback equivalence, and the global stabilization of minimum phase nonlinear systems," IEEE Trans. Automat. Contr., vol. 36, pp. 1228-1240, 1991.

[10] R. Lozano, B. Brogliato, and I. D. Landau, "Passivity and global stabilization of cascaded nonlinear systems," IEEE Trans. Automat. Contr., vol. 37, pp. 1386-1388, Sept. 1992.

[11] A. Bacciotti, "Potentially global stabilizability," IEEE Trans. Automat. Contr., vol. AC-31, pp. 974-976, Oct. 1986.

[12] A. Teel and L. Praly, "Tools for semi-global stabilization by partial state and output feedback," SIAM J. Contr. Opt., vol. 33, no. 5, pp. 1443-1488, Sept. 1995

[13] Z. Lin and A. Saberi, "Semi-global stabilization of partially linear composite systems via linear dynamic state feedback," in Proc. 32th Conf. Decision Contr., San Antonio, TX, Dec. 1993, pp. 2538-2543.

[14] R. H. Middleton, "Trade-offs in linear control system design," Automat ica, vol. 27, no. 2, pp. 281-292, 1991.

[15] J. H. Braslavsky and R. H. Middleton, "Global and semi-global stabilizability in certain cascade nonlinear systems," Dep. Electr. Comput. Eng., Univ. Newcastle, Tech. Rep. EE9340, Sept. 1993.

[16] P. Hartman, Ordinary Differential Equations. New York: Wiley, 1964

[17] M. Morari and E. Zafiriou, Robust Process Control. Englewood Cliffs, NJ: Prentice-Hall, 1989.

\section{Optimal Control of Manufacturing Flow and Preventive Maintenance}

\author{
E. K. Boukas and H. Yang
}

\begin{abstract}
In this paper, we address the optimal control issues corresponding to the planning of the production rate and maintenance rate of a failure prone manufacturing system. The discounted cost of our optimization problem is assumed to be a function of the inventory and the maintenance rate, where certain cost rates are specified for positive and negative inventories and maintenance, and there is a constant demand rate for the commodity produced. The objective of this paper is to choose the rates of production and maintenance to minimize a discounted cost over infinite horizon and to partially characterize the optimal solution. In the case where the maximum maintenance rate $\bar{v}$ is equal to a constant coefficient times the maximum production rate $\bar{u}$, we obtain a solution and show that the optimal solution is characterized by a critical surface, namely "hedging surface." Its description is also given.
\end{abstract}

\section{INTRODUCTION}

Manufacturing systems have received much attention recently. One way of dealing with manufacturing system problems is stochastic control method. One uses a special class of systems called piecewise deterministic systems to model this class of production system. See, for example, the work of Boukas [3], Boukas et al. [5], Davis [8], Rishel [9], Sworder [10], Vermes [11], and Wonham [12] for the description of this class of systems and their applications. In most of the manufacturing flow control models considered, the authors do not consider the machine age. Boukas and Haurie [4] give a model which permits the simultaneous planning of production and maintenance in a flexible manufacturing system where the probability of failure of

Manuscript received August 24, 1994; revised December 5, 1995. This work was supported by the Natural Sciences and Engineering Research Council of Canada under Grant OGP0036444.

The authors are with the Mechanical Engineering Department, École

Polytechnique de Montréal and the GERAD, Montréal, Quebec, Canada.

Publisher Item Identifier S 0018-9286(96)03722-1. 